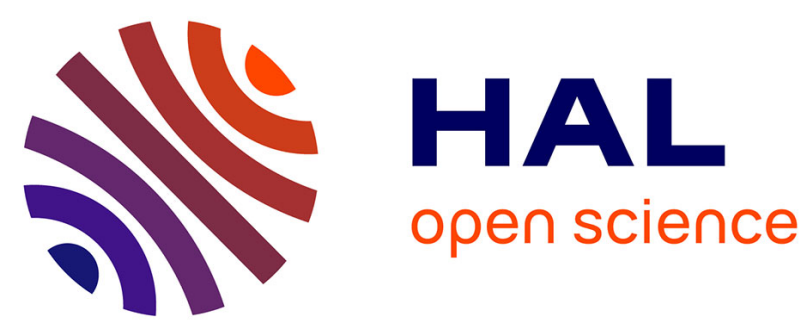

\title{
LE TAUREAU MARQUE SON TERRITOIRE (FESTIVITÉS TAURINES ET IDENTITÉS TERRITORIALES DU SUD-OUEST EUROPÉEN À L'AMÉRIQUE LATINE) \\ Jean-Baptiste Maudet
}

\section{To cite this version:}

Jean-Baptiste Maudet. LE TAUREAU MARQUE SON TERRITOIRE (FESTIVITÉS TAURINES ET IDENTITÉS TERRITORIALES DU SUD-OUEST EUROPÉEN À L'AMÉRIQUE LATINE). Annales de géographie, 2006, 650, pp.361-387. 10.3917/ag.650.0361 . halshs-01388915

\section{HAL Id: halshs-01388915 https://shs.hal.science/halshs-01388915}

Submitted on 27 Oct 2016

HAL is a multi-disciplinary open access archive for the deposit and dissemination of scientific research documents, whether they are published or not. The documents may come from teaching and research institutions in France or abroad, or from public or private research centers.
L'archive ouverte pluridisciplinaire HAL, est destinée au dépôt et à la diffusion de documents scientifiques de niveau recherche, publiés ou non, émanant des établissements d'enseignement et de recherche français ou étrangers, des laboratoires publics ou privés. 


\section{LE TAUREAU MARQUE SON TERRITOIRE (FESTIVITÉS TAURINES ET IDENTITÉS TERRITORIALES DU SUD-OUEST EUROPÉEN À L'AMÉRIQUE LATINE)}

Jean-Baptiste Maudet

Armand Colin | «Annales de géographie »

$2006 / 4 n^{\circ} 650 \mid$ pages 361 à 387

ISSN 0003-4010

ISBN 9782200920944

Article disponible en ligne à l'adresse :

http://www.cairn.info/revue-annales-de-geographie-2006-4-page-361.htm

\section{Pour citer cet article :}

Jean-Baptiste Maudet, « Le taureau marque son territoire (festivités taurines et identités territoriales du Sud-ouest européen à l'Amérique Latine) », Annales de géographie 2006/4 ( $\mathrm{n}^{\circ}$ 650), p. 361-387.

DOI 10.3917/ag.650.0361

Distribution électronique Cairn.info pour Armand Colin.

(C) Armand Colin. Tous droits réservés pour tous pays.

La reproduction ou représentation de cet article, notamment par photocopie, n'est autorisée que dans les limites des conditions générales d'utilisation du site ou, le cas échéant, des conditions générales de la licence souscrite par votre établissement. Toute autre reproduction ou représentation, en tout ou partie, sous quelque forme et de quelque manière que ce soit, est interdite sauf accord préalable et écrit de l'éditeur, en dehors des cas prévus par la législation en vigueur en France. Il est précisé que son stockage dans une base de données est également interdit. 


\title{
Le taureau marque son territoire (festivités taurines et identités territoriales du Sud-ouest européen à l'Amérique Latine)
}

\author{
When the bull marks its territory \\ (tauromachy and associated festivities in Southwestern \\ Europe and Latin America's regional identities)
}

\author{
Jean-Baptiste Maudet
}

Résumé La tauromachie, souvent confondue avec la seule corrida, regroupe en réalité une grande diversité de pratiques du Sud-Ouest européen à l'Amérique Latine. Qu'il s'agisse de la tauromachie professionnelle ou de la tauromachie participative, la fête et les taureaux semblent partout indissociablement liés. Cette relation qui mérite un examen critique peut être interprétée comme l'adaptation des fêtes taurines aux enjeux et au fonctionnement des sociétés contemporaines. Les divertissements taurins s'affichent alors comme de puissants marqueurs territoriaux à différentes échelles. La façon dont les diverses cultures taurines prennent formes et significations les unes par rapport aux autres permet d'identifier, de délimiter et de différencier une aire culturelle sous influence hispanique. Cette approche s'inscrit pleinement dans une réflexion plus générale sur la territorialité.

Resumen: La tauromaquia que suele ser confundida con la corrida de toros, abarca en realidad una gran variedad de prácticas desde el Suroeste de Europa hasta Latinoamérica. Tanto en la tauromaquia profesional como en los festejos populares, la fiesta y los toros parecen vincularse íntimamente. Esta relación, que supone un examen profundizado, puede ser interpretada como la adaptación de las fiestas taurinas a los resortes y modos de funcionamiento de las sociedades contemporáneas. Los toros aparecen como llamativos signos de identificación territorial que actúan a distintas escalas geográficas. Las relaciones entre las diferentes culturas taurinas permiten identificar, delimitar y diferenciar una zona cultural que expresa cierta influencia hispánica. Este enfoque se inserta en una reflexión más amplia sobre la territorialidad.

Abstract Often reduced to the better-known corrida de toros or bullfighting, tauromachy includes a great diversity of practices in Southwestern Europe and Latin America. Whatever are the tauromachy practices under consideration, professional or not, festivals and bulls are always associated in the stages of the regions. This systematic relation, which requires a cautious examination, can be interpreted as the tauromachy's adaptation to the stakes and functioning of contemporary societies. Moreover, on every stage tauromachy practices find a way to define and differenciate itself from the others. Tauromachy then acts as a powerful territorial marker on various geographical scales and contributes to identify, delimit and differentiate a cultural area under Hispanic influence. The chosen approach to study this phenomenon can be seen as being part of a more general investigation on territoriality.

Mots clés Tauromachie, corrida, identité, territoire, fête, Sud-Ouest européen, Amérique Latine.

Palabras Tauromaquia, corrida de toros, identidad, territorio, fiesta, Suroeste europeo, claves Latinoamérica.

Key-words Tauromachy, bullfighting, identity, territory, Southwestern Europe, Latin America.

Ann. Géo., $n^{\circ}$ 650, 2006, pages 361-387, (C) Armand Colin 


\section{Introduction}

Peu de tauromachie sans fête, peu de fête sans tauromachie. Dans les régions de tradition taurine, la tauromachie et la fête semblent indissociablement liées au point qu'elles finissent par être assimilées comme un seul et même phénomène de société : les fêtes taurines. Derrière cette évidence qui ne peut faire l'économie d'un examen critique se cachent des réalités sociales et géographiques très hétérogènes. Cette hétérogénéité tient d'une part à la nature même des phénomènes festifs qui exigent d'être qualifiés pour être significatifs et tient d'autre part à la diversité généralement méconnue des pratiques tauromachiques. La tauromachie est en effet un phénomène beaucoup plus diversifié que ne le laisse croire la véritable hégémonie médiatique exercée par la corrida espagnole. Un constat presque analogue pourrait être établi à propos des études en sciences sociales qui traitent des phénomènes taurins. Alors que les études sur la corrida sont nombreuses, les approches comparatives restent rares. Elles n'en sont que d'autant plus précieuses même si la dimension géographique des pratiques taurines demeure insuffisamment prise en compte. Cet article ne prétend pas pouvoir y remédier mais souhaite insister sur l'intérêt géographique de prendre en considération l'espace d'exercice de la tauromachie dans son ensemble, non par souci d'exhaustivité mais pour montrer que les fêtes taurines prennent en partie forme et signification les unes par rapport aux autres, du Sud Ouest européen à l'Amérique Latine.

Cette approche est à resituer dans le cadre d'un questionnement géographique de plus en plus attentif aux phénomènes festifs et d'une exigence à laquelle, comme le laisse entendre Guy Di Méo, la géographie ne doit sans doute pas renoncer. Dans son ouvrage La géographie en fête, il rappelle que les rares géographes qui se sont intéressés à la fête, ont davantage insisté sur le rôle de celle-ci dans la construction des identités locales que dans celles des identités régionales ou nationales ${ }^{1}$. L'enjeu n'est pas d'affirmer de façon systématique qu'il est du ressort de la géographie d'étudier la construction des identités aux échelles locales, régionales et nationales comme s'il s'agissait d'épuiser par strate le champ de l'explication. Il n'est pas non plus question de répéter de façon dogmatique que l'analyse multiscalaire peut apporter aux explications en sciences sociales une spécificité propre à la géographie, face aux approches sociologiques et anthropologiques de la fêtte. L'objectif est d'évaluer dans quelle mesure l'identité locale prend sens en fonction d'un contexte spatial plus englobant et jusqu'à quel point les niveaux d'identification territoriale fonctionnent ensemble. Nous faisons l'hypothèse que l'analyse des fêtes taurines offre un terrain fertile pour réfléchir dans cette perspective au fonctionnement de la territorialité au sein d'une aire culturelle que la tauromachie contribue à identifier, délimiter et différencier. Pour cela nous présenterons l'emprise spatiale des pratiques tauromachiques, nous analyserons les relations

1 Di Méo, 2001, p. 1. 
complexes qui unissent la fête et la tauromachie dans l'espace et dans le temps, puis nous évaluerons le rôle et la place de la tauromachie dans les processus de territorialisation à différentes échelles.

Corrida

Course landaise

Course camarguaise

Charreada

Coleo, vaquejada

Course de recortadores

Jaripeo professionnel

Rodéo chilien

Rodéo nord américain

Lieux spécialisés

Tauromachies professionnelles

Lieux non spécialisés

Capeia raiana

Corralejas

\begin{tabular}{|ll} 
& $\begin{array}{l}\text { Divers lâchers de bovins } \\
\text { Bous de carrer } \\
\\
\text { Touradas a corda } \\
\text { Yawar fiesta } \\
\text { Encierros } \\
\text { Jaripeo populaire }\end{array}$ \\
\hline
\end{tabular}

Fig. 1 Diagramme de différenciation des pratiques tauromachiques.

Chart of the tauromachy practices.

\section{La diversité tauromachique et son emprise spatiale}

\subsection{La tauromachie entre spectacle professionnel et pratique participative}

En tant que phénomène social et géographique, la tauromachie est un divertissement fondé sur un affrontement entre des hommes et des bovins. Dans le registre des divertissements contemporains, la tauromachie procède de deux réalités sociologiques différentes. D'une part, la tauromachie est un spectacle professionnel qui se déroule dans une enceinte spécifiquement construite à cet effet dont les arènes constituent l'équipement le plus représentatif. D'autre part, la tauromachie est une pratique participative qui investit un espace public plus ou moins aménagé pour l'occasion. Par tauromachie professionnelle, il faut entendre toutes les pratiques où il existe 
une frontière stricte entre les acteurs spécialistes du jeu taurin et les spectateurs, et par tauromachie participative, celles où sont confondus acteurs et spectateurs. Il existe une forte corrélation entre le degré de professionnalisation de la tauromachie et le degré de spécialisation des lieux de sise en scène (voir diagramme et photographies).

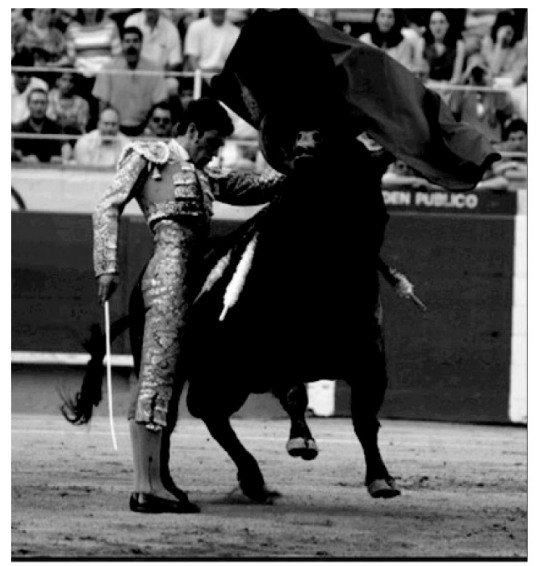

Corrida

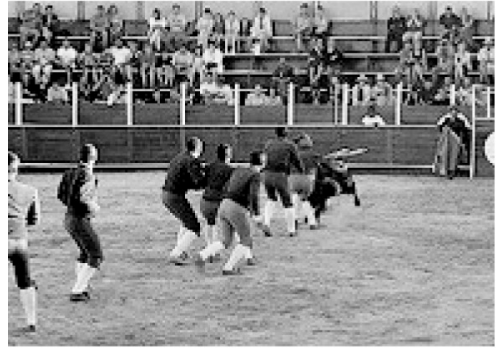

Tourada (forcados)

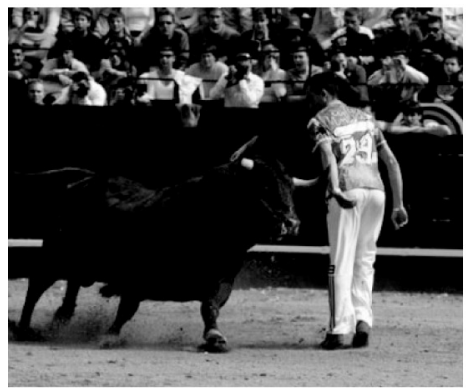

Concours de recortadores

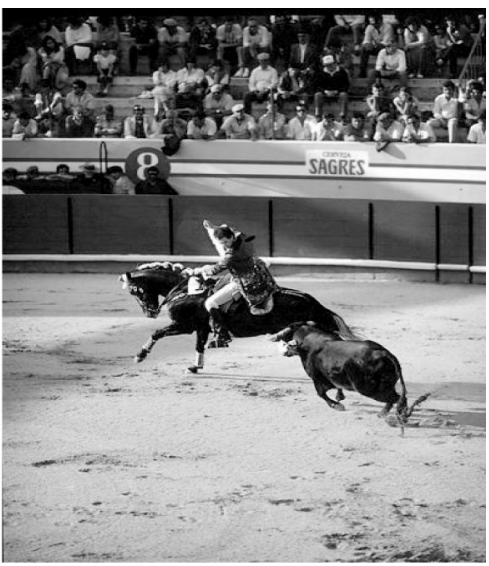

Tourada ou corrida portugaise (cavalier)

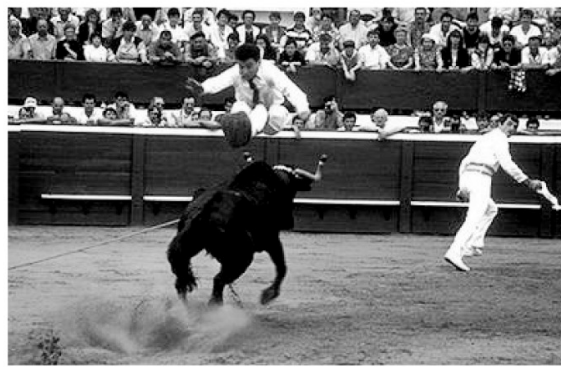

Course landaise

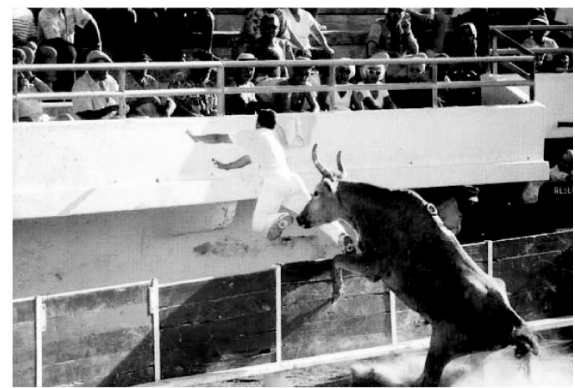

Course camarguaise

Fig. 2 La diversité des pratiques tauromachiques.

Diversity in the tauromachy practices. 


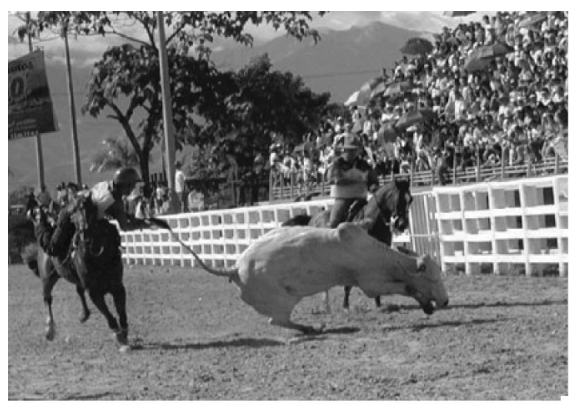

Coleo

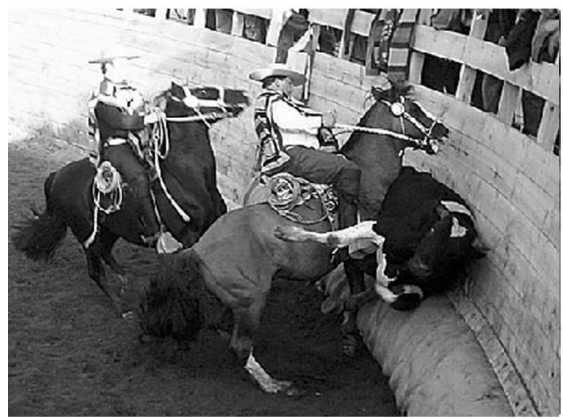

Rodéo chilien

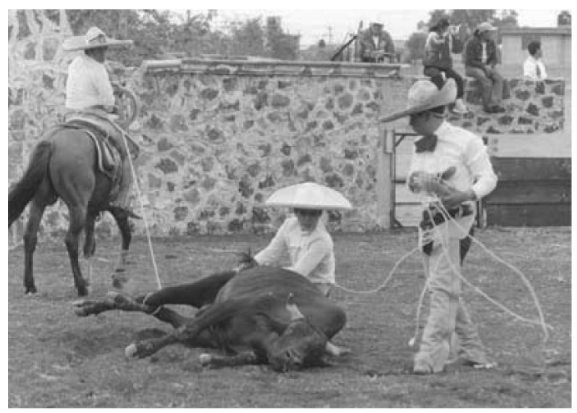

Charreada (terna en el ruedo)

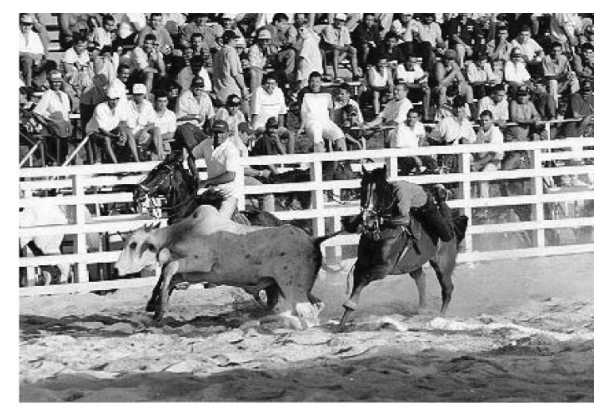

Vaquejada

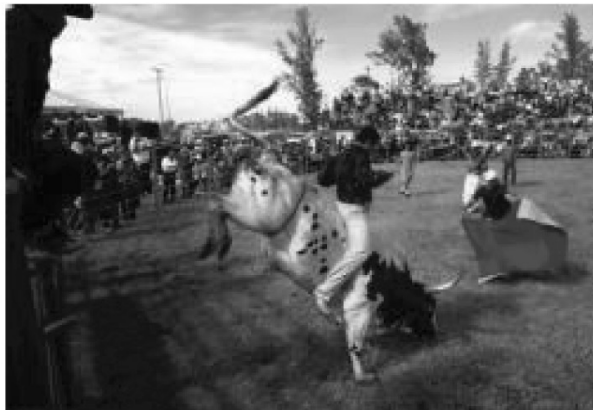

Jaripeo populaire

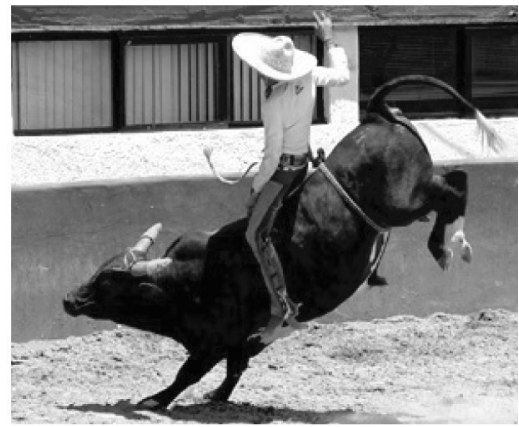

Charreada (jineteo)

Les tauromachies professionnelles sont à la croisée de deux types de spectacles modernes : le sport et la représentation artistique. La course landaise, la course camarguaise, le coleo, la charreada, la vaquejada, la rodéo chilien, le rodéo nord américain peuvent être rapprochés du modèle sportif. Les saisons sont structurées sur la base de championnats annuels qui peuvent être subdivisés en compétitions locales, régionales ou nationales, reliées entre elles par un système de notation qui permet de classer sur l'année la performance des protagonistes. La corrida est plutôt à rapprocher du modèle de la représentation artistique puisque l'évaluation de la performance est plus qu'ailleurs subordonnée aux critères subjectifs de l'interprétation. De plus, il n'existe ni 


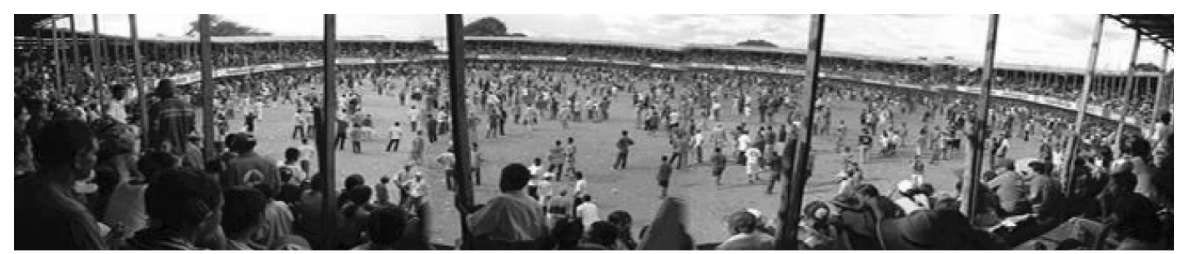

Fiesta en corraleja (Colombie)
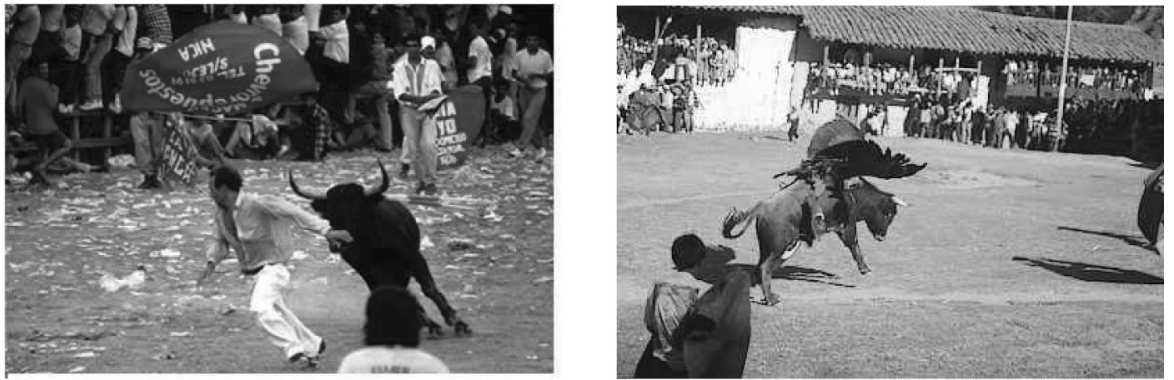

Corraleja (Colombie)

Yawar fiesta ou turupukllay (Pérou)
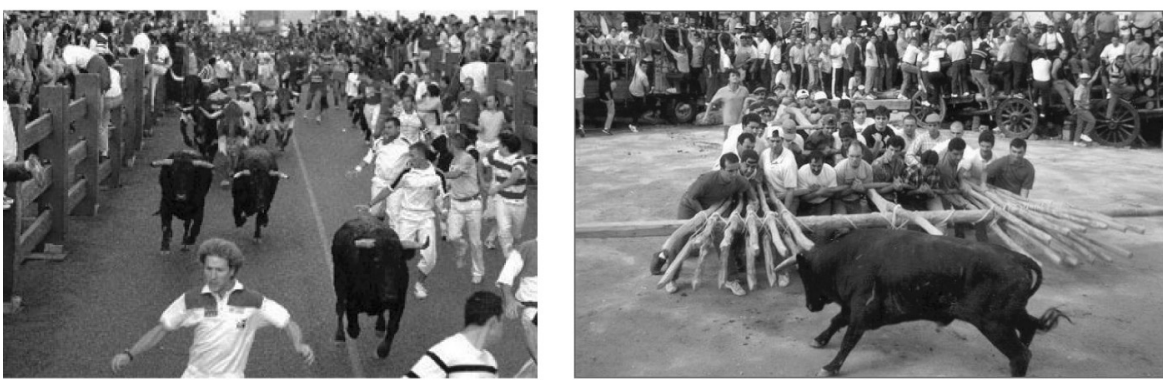

Encierro (Espagne)

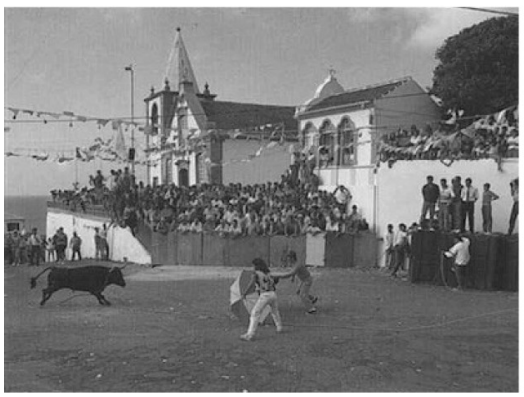

Capeia raiana (Portugal)

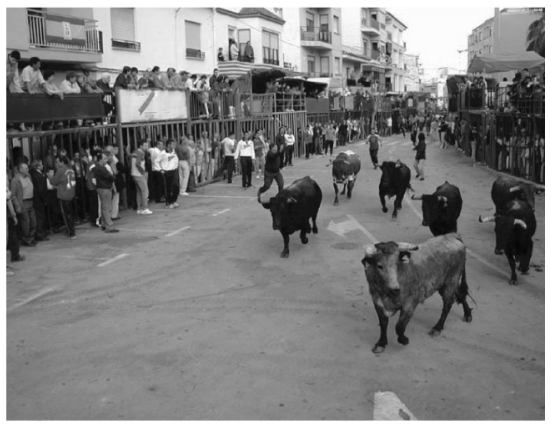

Tourada a corda (Açores-Portugal)

Bous al carrer (Valence-Espagne)

championnat ni classement officiel établi par une quelconque entité régulatrice. Pour qu'il n'y ait aucune équivoque sur une impossible hiérarchie entre ce qui relèverait de l'art et ce qui relèverait du sport, précisons que 
l'ensemble des spectateurs sont autant attentifs, si ce n'est plus, au style des protagonistes qu'aux décisions du jury, de sorte que les catégories sont en réalité perméables. Comme le souligne Bernard Traimond à propos de la course landaise et de la course camarguaise : "Ces jeux se trouvent entre deux dynamiques contradictoires, l'une qui leur fait adopter les formes et les valeurs du sport, l'autre qui les refuse. ${ }^{2}$. À l'inverse, les tauromachies participatives ne désignent presque jamais de vainqueur même s'il est toujours question de faire remarquer son adresse et son courage, pour la gloire qui peut parfois s'accommoder de récompenses matérielles.

\subsection{L'emprise spatiale des pratiques tauromachiques}

Comme le montre les cartes 1 et 2 ci-dessous, les pratiques tauromachiques ont des aires d'extension très variables dont certaines se recoupent partiellement. La corrida est présente dans huit pays situés de part et d'autre de l'Atlantique : l'Espagne, la France, le Portugal, le Mexique, le Venezuela, la Colombie, l'Équateur et le Pérou. Elle est pratiquée partout selon les mêmes règles et le même cérémonial sauf au Portugal où la mise à mort publique du taureau est interdite par la loi. L'aire d'extension de la corrida espagnole contraste avec celle de la corrida portugaise (ou tourada) qui n'est guère pratiquée ailleurs qu'au Portugal ${ }^{3}$. Ce fut pourtant le cas au Brésil, à Macao puis en Angola et au Mozambique, à l'époque où le pays était une puissance coloniale. Corrida espagnole et corrida portugaise sont donc deux formes tauromachiques transnationales d'origine européenne qui ont connu un destin divergent. La première est devenue une forme dominante, fortement enracinée dans une grande partie de l'aire culturelle hispanique, la seconde, par un cheminement inverse, s'est repliée en métropole et s'est vue marginalisée dans le reste de l'aire culturelle lusophone.

D'autres tauromachies s'étendent sur plusieurs pays. C'est le cas par exemple du rodéo dans sa version nord américaine, pratiqué aux États-Unis, au Canada mais aussi au Mexique et au Brésil. Il est intéressant de remarquer que les techniques du rodéo nord américain, aujourd'hui fortement identifiées à la culture de l'Ouest des États-Unis, sont originaires du Mexique dont la frontière s'est déplacée vers le Sud ${ }^{4}$. Au Mexique, ces techniques sont à la base de la charreada qui est pratiquée dans tout le pays ainsi que dans les régions états-uniennes où s'est établie une importante communauté mexicaine. Les aires des pratiques se chevauchent mutuellement sur une des frontières politiques, économiques et culturelles les plus tangibles du continent américain. Le coleo offre un autre exemple de tauromachie transfrontalière. Son foyer principal correspond à la région des llanos, grande plaine de savane tropicale

2 Traimond, 1996, p. 104.

3 Pour une approche comparée de la géographie des pratiques taurines portugaises et espagnoles voir Maudet, 2006.

4 Saumade, 2005. Nous tenons l'essentiel des informations sur les jeux taurins mexicains et en particulier sur la charreada et le jaripeo d'un document privé de Frédéric Saumade sans lequel cet article n'aurait pu être mené à bien. Qu'il en soit remercié à la hauteur de ses qualités humaines et scientifiques. 
située en rive gauche de l'Orénoque, partagée entre le Venezuela et la Colombie. En réalité, la technique du coleo qui consiste à faire chuter le taureau en l'attrapant par la queue, est employée ailleurs, au Brésil dans les vaquejadas du Nordeste et au Mexique où elle fait partie des multiples exercices de la charreada. On peut remarquer que seul le rodéo chilien, bien qu'il s'agisse également d'un jeu taurin issu du maniement du grand bétail dans les élevages extensifs, ne partage sa technique avec aucune autre pratique.

Les rodéos et la corrida sont deux formats tauromachiques qui répondent à des logiques d'organisation différentes. La corrida ne connaît pas de véritables frontières à l'intérieure de son aire d'extension (à l'exception du Portugal) alors que les rodéos dépendent pour la plupart d'organisations territorialisées correspondant aux divisions administratives à l'instar de nombreux sports. La corrida relève fondamentalement d'une pratique transatlantique et transnationale d'origine espagnol alors que les spectacles de rodéo relèveraient davantage d'une pratique paraméricaine fragmentée par le cadre des États Nations. Il existe néanmoins une internationalisation du coleo ainsi que du rodéo dans sa version nord-américaine.

Enfin, on observe des pratiques tauromachiques d'extension plus réduite. Elles offrent un éventail diversifié de pratiques codifiées, parfaitement identifiables et spatialement délimitées: la capeia raiana, la course camarguaise et la course landaise dont les aires d'extension recoupent très imparfaitement les Landes et la Camargue. En Espagne, le bous de carrer s'étend sur les provinces du Levant (Alicante, Valence et surtout Castellon) auxquelles on peut joindre l'embouchure de l'Èbre située en Catalogne. Les concours de recortadores ont lieu majoritairement dans la partie septentrionale et orientale du pays mais ils connaissent un succès croissant dans toute la Castille. En Amérique Latine, il faut signaler la pratique des fiestas en corralejas sur la côte caraïbe de la Colombie (départements de Sucre, Córdoba, Bolivar) et celle du yawar fiesta ou turupukllay dans les Andes du Sud péruvien. Enfin, on observe dans tous les pays de tradition taurine de très nombreuses façons de "courir les taureaux », divers encierros à pied ou à cheval et divers lâchers de bovins. Dans le cas de l'Espagne, pour environ 1500 spectacles taurins du haut de la hiérarchie par an (corridas et novilladas piquées), on compte aujourd'hui autour de 15000 festejos taurinos populares ${ }^{5}$.

\subsection{Tauromachie des villes, tauromachie des champs}

Dans les pays de tradition taurine, la corrida apparaît volontiers comme le spectacle représentatif de la ville et d'une certaine urbanité quand les autres pratiques sont plutôt l'apanage des petites villes et villages du monde rural. En Espagne, la représentation dominante du fait taurin considère souvent ces dernières comme des formes archaïques s'opposant à la corrida qui serait la forme moderne, savante et civilisée de la tauromachie, quelle que soit sa localisation.

5 Les festejos taurinos populares désignent en Espagne les tauromachies non professionnelles à caractère participatif. 


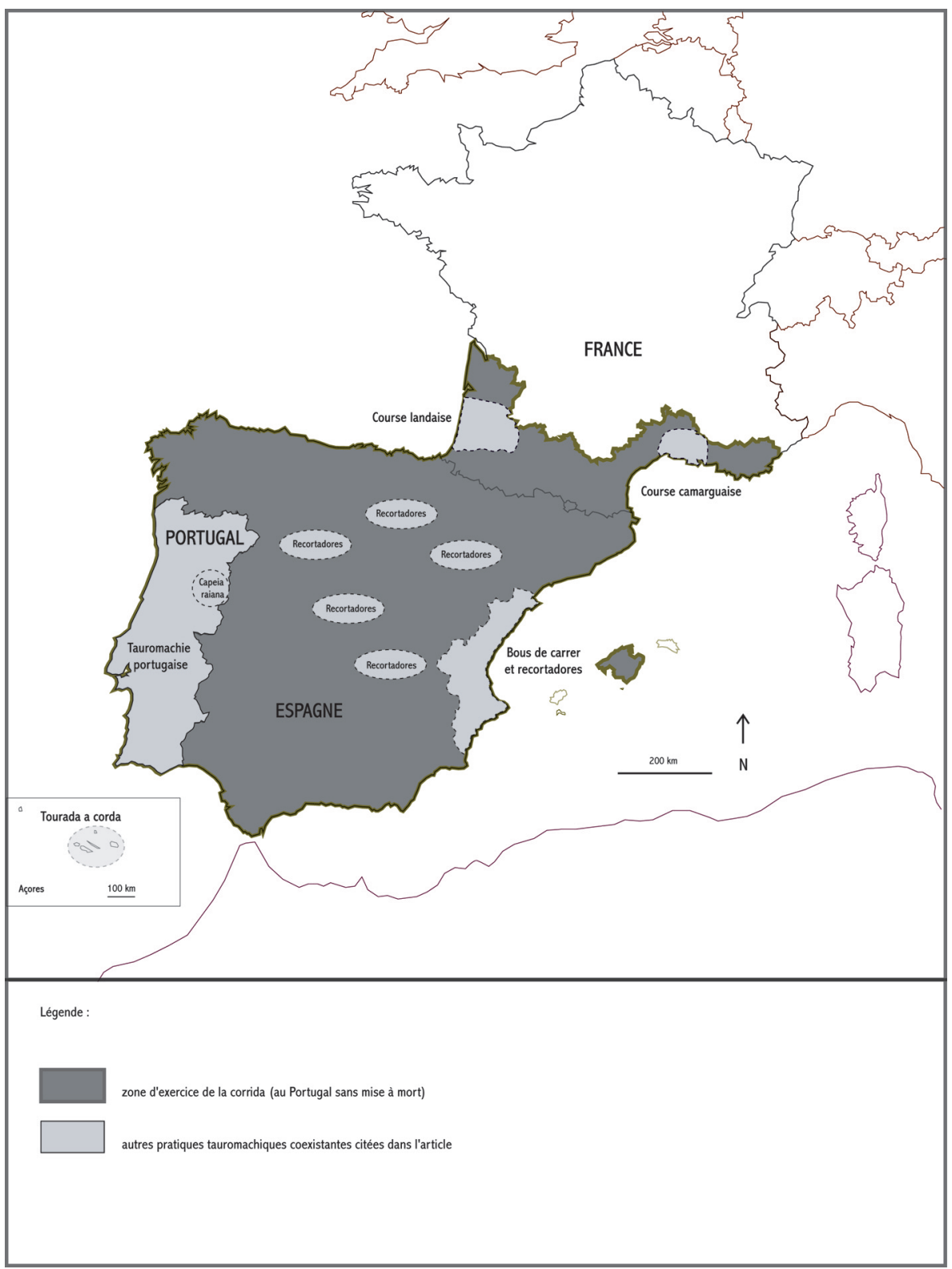

Carte 1 Les pratiques tauromachiques dans le Sud-Ouest européen. Tauromachy pratices in Southwestern Europe. 


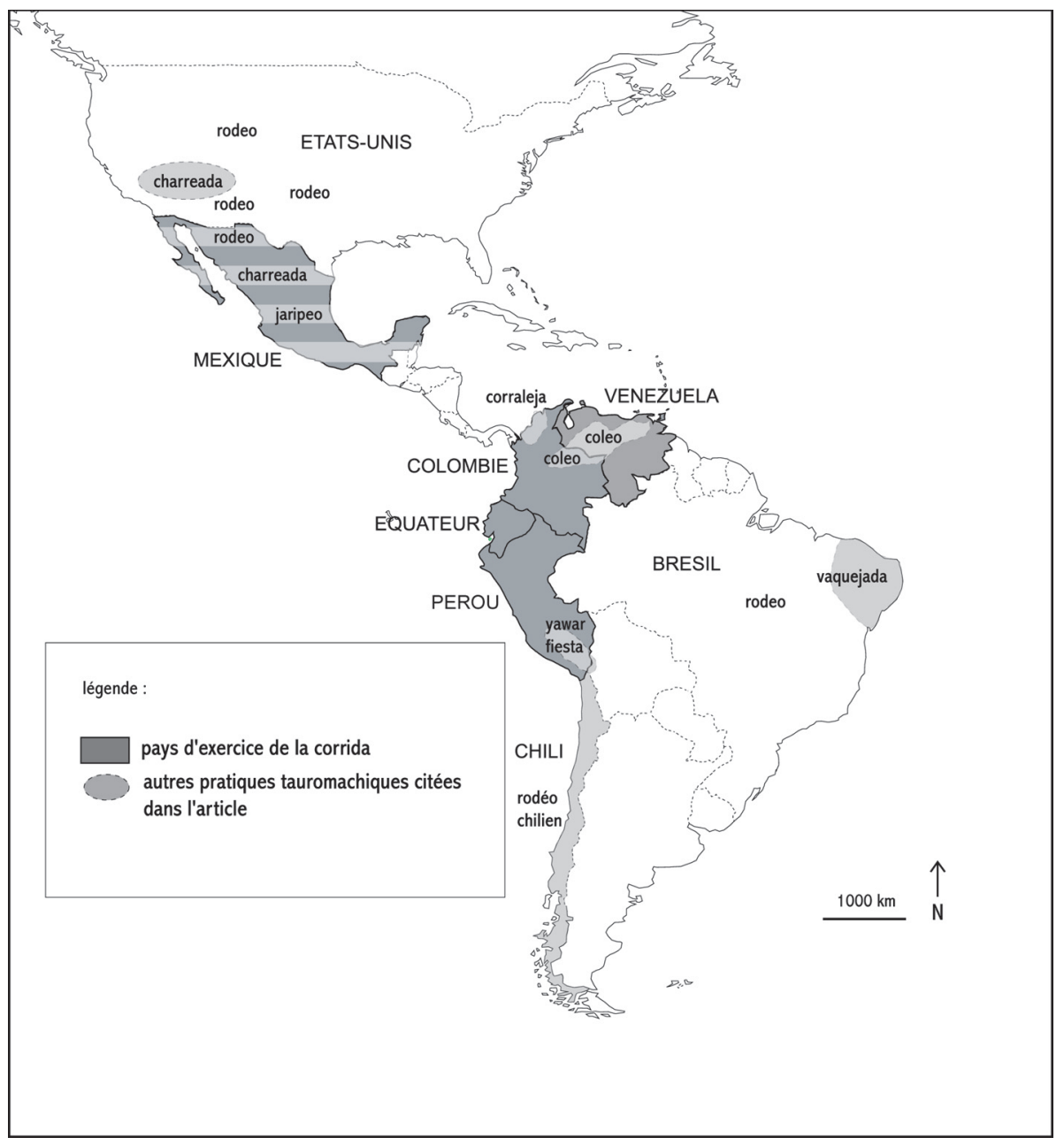

Carte 2 Les pratiques tauromachiques en Amérique Latine.

Tauromachy pratices in Latin America.

Ceci étant, toutes les pratiques tauromachiques s'appuient plus ou moins explicitement sur une emblématique rurale. Dans une certaine mesure, le milieu rural apparaît comme la terre d'élection et le cadre naturel de la tauromachie. Même si les finales des grandes compétitions de courses camarguaises ont lieu généralement dans les arènes de Nîmes et d'Arles, les hauts lieux de la géographie symbolique de la fe di biòn 6 sont des arènes du monde rural comme Lunel, Beaucaire, Marsillargues, Saint-Laurent d'Aigouze ou encore Le

6 La fe di biòu désigne en Camargue la passion des taureaux et des courses. 
Cailar, situé au milieu des élevages de taureaux et baptisé «la Mecque de la course camarguaise ». Il en est de même pour la course landaise qui possède son musée dans le village de Bascons et sa " Mecque » aux arènes de Pomarez en Chalosse. Le caractère rural des pratiques tauromachiques apparaît également dans les modalités même de certains jeux taurins qui s'inspirent des méthodes traditionnelles de domestication du bétail. Le meilleur exemple est sans doute l'utilisation récurrente de la corde que l'on retrouve sur toute la géographie taurine : les touradas a corda de l'île Terceira des Açores, les vacas de corda de l'Alto Minho, les sokamuturra du Pays basque, la course landaise, le taureau à la bourgine camarguais, la reata (lasso) de la charreada. Il en va de même de la figure du vacher à cheval, transposée dans la sphère ludique par la tauromachie : abrivado et bandido camarguais, encierro à cheval de Segorbe (Castellon), picarias a vara larga au Portugal, vaquejada, coleo, charreada, cavaliers des fiestas en corralejas.

Le caractère rural n'est pas absent de l'univers de la corrida comme le prouve la fascination exercée par les élevages de taureaux et par le campo, véritable antichambre de la culture taurine ${ }^{7}$. Pourtant, c'est bien la grande ville qui consacre le succès du spectacle et qui établit les hiérarchies: Madrid, Séville, Bilbao, Valence, Pampelune, Mexico, Bogota, Cali, Quito, Lima. On remarque qu'en France, les cités taurines les plus renommées (Nîmes, Arles, Béziers, Bayonne, Dax, Mont-de-Marsan, Vic-Fezensac), ne correspondent pas aux métropoles régionales de la France du Sud (Bordeaux, Toulouse, Montpellier ou Marseille). Ces villes ont pourtant connu de riches heures tauromachiques avec lesquelles certaines tentent aujourd'hui de renouer (les arènes de Floirac pour Bordeaux, celles de Fenouillet pour Toulouse). Retenons que pour l'ensemble des pratiques taurines, tout se passe comme si la ruralité et le lien avec les sociétés agraires étaient garants d'une certaine authenticité des valeurs tauromachiques à laquelle la ville ne peut pas complètement prétendre.

\section{La Fête et la tauromachie}

\subsection{Festivités taurines ou tauromachies festives}

Il existe un lien particulièrement fort entre la fête et la tauromachie. Dans les régions taurines, il est rare en effet que la tauromachie ne soit pas partie prenante des réjouissances festives des calendriers civils ou religieux au point que fêtes et taureaux ont tendance à être assimilés. Dans les pays hispanophones, cette relation se voit renforcée par un large champ sémantique qui les associe. Pour l'aficionado, le mot fiesta suffit, à lui seul, à designer la tauromachie. On le retrouve dans fiesta taurina, fiesta de los toros, festejos taurinos, fiesta brava. Autant d'emplois courants, propre à la culture taurine, qui désignent la tauromachie elle-même et l'univers culturel dans

7 Le campo désigne à la fois le champ et la campagne. 
lequel elle s'inscrit. Mieux, selon le dictionnaire de l'Académie Royale Espagnole, la corrida de toros est une "fête qui consiste à combattre un certain nombre de taureaux sur une place fermée ${ }^{8}$. Il est symptomatique que le terme de spectacle ne lui ait pas été préféré. On retrouve cet usage de l'autre côté de l'Atlantique : fiesta en corralejas, fiesta charra, fiesta del coleo ou fiesta llanera. La langue française semble moins marquée par cette assimilation quasi systématique mais la réalité sociologique est bien la même : la fête et la tauromachie semblent indissociablement liées.

Un exemple très concret en donne la mesure, celui de l'annulation de la corrida de la féria des Vendanges de Nîmes en raison des événements du 11 septembre 2001, survenus le mardi précédent à New York. Rappelons que le mot féria est la transposition directe du mot espagnol feria (la foire) qui désigne, dans le contexte taurin, un ensemble de courses de taureaux programmé à l'occasion d'une fête. Cette acception est valable en péninsule Ibérique, en France et en Amérique latine. Mais en France, la féria est par définition taurine, ce qui n'est pas le cas ailleurs. Suivons dans ces grandes lignes les propos rapportés par le chroniqueur Manolillo dans la revue Toros $^{9}$. Après deux jours de palabres, la décision de l'annulation de la corrida et de toutes les festivités du vendredi est finalement prise par les élus locaux et les organisateurs de la féria des Vendanges :

Le trio fête-féria-corrida est en place, avec comme conséquence que les trois forment un ensemble indistinct dont il ressort aujourd'hui que la corrida est assimilée à une fête. [...] La regrettable association féria-fêtecorrida a finalement pris le dessus. Il ne pouvait être question de laisser se dérouler une corrida-fête un jour de deuil national. Ce fut donc l'annulation de tout ${ }^{10}$.

Il n'est pas certain que cette association soit aussi regrettable pour les aficionados car elle contribue aussi à la vitalité de la tauromachie. Cependant, reconnaissons qu'il n'est pas toujours facile de savoir si la tauromachie est la cause ou la conséquence de la fête, tant les logiques festives les imbriquent jusqu'à faire disparaître l'objet même de ce qui est fêté au profit de ces manifestations plurielles. Cet objet, quand il est possible de l'identifier au-delà des effets d'annonce, peut être central pour les uns, marginal pour les autres, absent pour les troisièmes, sans que ne soit jamais remise en cause la loi selon laquelle la fête engendre et attire la fête.

\subsection{La tauromachie, la fête et le spectacle}

Il existe une imbrication complexe entre la fête et le spectacle taurin. En péninsule Ibérique, à partir du $\mathrm{XI}^{\mathrm{e}}$ siècle, la noblesse prend l'habitude de donner des courses de taureaux en place publique pour célébrer les naissances, les baptêmes, les mariages princiers ou la canonisation d'un saint.

8 Real Academia Española, Diccionario de la lengua española, 2001, XXII édition, p. 450.

9 Toros, $\mathrm{n}^{\circ} 1662,27$ septembre 2001, p. 1.

10 Ibid p. 1 
La Renaissance entretient à merveille une ambiguïté baroque où la fête est un spectacle et le spectacle, une fête. Le peuple possède aussi ces propres jeux où l'on court les taureaux à l'occasion des noces ou des fêtes votives. $\mathrm{Au} \mathrm{XIX}^{\mathrm{e}}$ siècle, les spectacles taurins se greffent aux foires commerciales, les ferias, en particulier les foires aux bestiaux comme celle de Séville officialisée en 1846. Parmi les événements programmés de la fête officielle ou les distractions de la foire commerciale, le divertissement taurin apparaît comme le grand moment d'effervescence communautaire au point qu'il accapare les attributs distinctifs de la fête : le rassemblement, le tumulte, l'enthousiasme. Aujourd'hui encore, on observe une coïncidence remarquable de la tauromachie avec la célébration des fêtes du calendrier civil et religieux malgré une incontestable autonomie du spectacle moderne qui sécrète aussi ces propres rendez-vous en marge des festivités traditionnelles. Quelques exemples permettent de montrer cette imbrication.

Les principales fêtes de Madrid sont celles données en l'honneur du saint patron Isidore Laboureur autour du 15 mai. L'un des divertissements majeurs des fêtes madrilènes est la féria taurine crée en 1948 par Livinio Stuyck alors imprésario des arènes de Madrid. À l'origine, la "feria de Madrid» ne fait que réunir sur cinq jours, à partir du 15 mai, les corridas normalement programmées sur tout le mois. Aujourd'hui, ce qu'il est convenu d'appeler la San Isidro est la plus longue et la plus importante féria du monde qui dure plus de trois semaines. Pour autant, elle n'a rien de festive. Aucune ambiance de fête dans les arènes qui préfèrent jouer le rôle d'un tribunal de l'orthodoxie taurine, aucune musique pendant que l'on torée contrairement aux autres arènes et aucun lien entre le spectacle taurin et d'autres lieux de fêtes. La féria relève d'une industrie culturelle dont la coïncidence avec la célébration du saint patron madrilène apparaît comme un prétexte lointain. Une analyse tout à fait comparable pourrait être menée à propos de la "feria taurina del Señor de los Milagros » à Lima, crée en 1946 sous le nom de "feria de octubre».

À l'inverse, certaines férias donnent l'impression que la fête urbaine surgit de la tauromachie elle-même. C'est le cas à Pampelune où la célébration de la San Fermin et la féria taurine (rebaptisée en 1959 la feria del toro) ont tendance à ne faire plus qu'un. En effet, l'espace et le temps des fêtes de la San Fermin semblent rythmés par la présence du taureau au cour de la ville: l'encierro à 8 heures qui conduit les taureaux jusqu'aux arènes, suivi d'un lâcher de vaches, puis la corrida en fin d'aprèsmidi où s'anime un public en liesse dans la partie "soleil » des gradins. L'articulation de la tauromachie professionnelle et de la tauromachie participative contribue à faire des sanfermines un "phénomène social total ".

Les fêtes andalouses offrent encore un autre modèle. À Séville, la feria de abril ne doit pas être confondue ave la féria taurine qui bien que située au cour du dispositif festif n'est qu'une des composantes de la fête urbaine. La fête, celle où l'on danse les sévillanes, où l'on chante, où l'on boit du vin de manzanilla à toute heure du jour et de la nuit, se tient d'ailleurs, 
depuis 1973, à l'extérieur du centre-ville dans le campo de féria du quartier de Los Remedios, un champ de foire de quelque $40000 \mathrm{~m}^{2}$. L'empreinte de la culture taurine y est omniprésente, les rues portent des noms de toreros andalous mais la tauromachie ne peut pas être considérée comme la fête elle-même. En tant que spectacle ordonné et réglementé, la tauromachie professionnelle pourrait même apparaître comme anti-festive comparée aux tauromachies participatives, dont le désordre et l'effervescence correspondent davantage à l'idée de "faire la fête ». On mesure clairement le contraste qu'il y a entre une corrida à Madrid et les fiestas en corralejas de Sincelejo, où environ deux milles personnes «courent» jusqu'à quarante taureaux par jour, dans le chaos le plus total, à pied et à cheval, au sein d'une arène de 365 mètres de diamètre, devant des milliers de spectateurs faisant la fête sur la piste et dans les gradins.

\subsection{Mesurer l'importance des fêtes taurines}

Sur notre espace, l'ensemble de la hiérarchie du peuplement est généralement concerné par les divertissements taurins, depuis les plus grandes villes jusqu'aux plus petits villages. L'espace taurin fonctionne comme un espace hiérarchisé où chaque lieu de mise en scène tauromachique, de l'arène à la place publique, est le centre d'un arrière pays taurin plus ou moins vaste. Par analogie avec la théorie des lieux centraux, les arènes ont pour fonction de distribuer un service très spécialisé : le divertissement taurin. Les arènes les plus importantes, celles des grandes villes, peu nombreuses, plus espacées, programment des spectacles professionnels du haut de la hiérarchie (généralement regroupés en férias pour ce qui est des corridas), destinés à des seuils de populations élevés et des aires d'influences élargies. Les arènes de rang inférieur, plus petites, s'adressent à des seuils de population plus réduits, des aires d'influences moins larges et programment des divertissements moins coûteux : novilladas, tauromachies régionales, tauromachies participatives. Il existe pour la tauromachie une sorte de loi rang-taille qui fait correspondre la hiérarchie des arènes et des divertissements taurins à celle de la trame du peuplement ${ }^{11}$. Cette organisation connait bien évidemment des variations régionales importantes en fonction de l'intensité de la culture taurine.

Cependant, l'importance des festivités taurines telle qu'elle est vécue par ses adeptes est en partie indépendante de ces hiérarchies. Avec un ou deux «jours de taureaux» dans l'année, la tauromachie peut être omniprésente dans la vie de la cité car c'est une occasion unique d'être rassemblé. On comprend mieux l'attachement de nombreux villages à leur fête taurine qui manifeste une adhésion à la culture taurine et exprime une certaine cohésion sociale. Cet attachement est d'autant plus fort que la

11 L'intérêt que constitue la théorie des lieux centraux pour analyser la hiérarchie et la localisation des équipements sportifs, souligné par Jean Pierre Augustin (1995) se vérifie pour I'étude des lieux de mise en scène tauromachique. 
fête taurine est unique en son genre comme le carnaval du taureau à Ciudad Rodrigo (Salamanca), le toro de la vega à Tordesillas (Valladolid), le toro de San Marco à Beas de Segura (Jaén), le toro de jubilo à Medinaceli (Soria) ou la corrida de morte à Barrancos (Alentejo). À l'autre bout de la hiérarchie du peuplement, quelle importance accorder à la culture taurine dans les grandes villes lorsque le spectacle taurin n'est qu'un divertissement parmi d'autres et que sa visibilité se retrouve noyée par le gigantisme urbain ? On ne peut mesurer l'importance des fêtes taurines aux yeux des sociétés qui les produisent sans garder en tête la taille des cités et l'emprise spatiale concrète de la fête.

De ce point de vue là, les festivités taurines ont sans doute le plus grand rôle là où la tauromachie est identifiée comme la raison d'être de la fête et du rassemblement, son noyau dur, son facteur coagulant. L'estimation de cette relation reste délicate et le plus souvent empirique. Elle est d'autant plus remarquable lorsqu'il s'agit d'une ville relativement importante comme Pampelune ou Nîmes où la transfiguration du temps et de l'espace par la fête taurine semble totale. En 2001, Jocelyne Pezet, conseillère régionale du Languedoc-Rousillon et conseillère municipale de Nîmes, évaluait à près d'un million de visiteurs le nombre de personnes attirées par la féria de Nîmes, soit sept fois la population de la ville ${ }^{12}$. D'après les résultats d'institut de sondage, à la question "pourquoi venez-vous à la féria ? », 16,8\% répondent pour les corridas et les manifestations taurines, 8,9\% pour les chevaux et le folklore, 49,5\% pour l'ambiance des fêtes, 24,8\% pour d'autres raisons. Par ailleurs, le Bureau d'Information et Prévention Économique estime que les dépenses cumulées d'environ 100000 spectateurs taurins lors de la féria s'élèvent à 13 millions d'euros et Jocelyne Pezet d'ajouter « la féria est une des rares manifestations de cette envergure qui coûte à la collectivité moins de $1 \%$ du flux économique obtenu ». On comprend l'engouement suscité par les fêtes taurines auprès de nombreuses municipalités en matière de développement local.

\section{$3 \quad$ Les festivités taurines : la tradition modernisée}

\subsection{Ruptures et continuités des fêtes du taureau}

L'histoire de la tauromachie produite pendant longtemps par des érudits aficionados plutôt que par des historiens de métier, a généralement privilégié une approche évolutionniste des festivités taurines. Les tauromachies contemporaines seraient la dernière étape d'un long processus de transformation qui remonte à la première rencontre entre les hommes et les bovins. Cette historiographie insiste assez lourdement sur l'ancestralité de la tauromachie qui lui confèrerait un surplus évident de légitimité. Il y aurait une

12 Information transmise lors du IV congrès mondial des villes taurines, septembre 2001, Vila Franca de Xira, Portugal. 
permanence à travers les âges de la posture tauromachique qui suffirait à démontrer la continuité positive des pratiques. Il n'est pas rare que soient alors mis en perspective, les chasses préhistoriques, le bestiaire taurin de l'iconographie paléolithique et néolithique ainsi qu'un hypothétique culte du taureau en Méditerranée qui aurait migré du croissant fertile à l'Espagne en passant par la Crète et la Rome antique. Cet héritage recentré sur la péninsule Ibérique s'épanouit ensuite à partir de la Reconquête lors des tournois et des fêtes royales qui célèbrent les grands événements de la couronne et de l'Église. Puis à partir de l'avènement des Bourbons sur le trône d'Espagne, la noblesse se désintéresse de la tauromachie et le peuple s'en empare pour l'élever au rang de fiesta nacional au XIX ${ }^{\mathrm{e}}$ siècle. La corrida est alors conçue comme le modèle festif taurin de référence qui a su sauvegarder les valeurs de la relation ancestrale entre l'homme et l'animal, conserver l'esprit d'effervescence communautaire autour du sens de l'honneur, sublimer en art majeur la tauromachie professionnelle et l'exporter dans le monde. La portée apologétique d'une telle reconstruction ne peut nous échapper. Ce discours d'abord conçu pour la corrida a servi de trame narrative aux autres pratiques tauromachiques du Sud-Ouest européen et de l'Amérique Latine dont les différences n'ont souvent été envisagées que comme la valorisation autochtone d'un patrimoine ancestral commun : le taureau est un animal sacré et la fête taurine surgit comme "recours au sacré », pour reprendre une expression de Caillois ${ }^{13}$.

Cette historiographie est largement critiquée à partir des années 1980, par un courant historique et anthropologique qui insiste au contraire sur les lacunes de l'histoire taurine et sur la nécessité de relativiser le modèle dominant par une approche comparée des pratiques ${ }^{14}$. Au regard de ces travaux, il semble impossible de prouver une seule et même origine à l'ensemble des pratiques tauromachiques. Il est tout au plus envisageable de considérer que chacune d'elle hérite dans des proportions variables de l'élevage (le maniement du bétail et son abattage), de la pratique militaire (les entraînements et les démonstrations de prestige de la noblesse), de la pratique religieuse (les rites et sacrifices propitiatoires) et de la pratique ludique, autonome ou dérivée des précédentes. De plus, les ruptures dans l'évolution de la tauromachie sont suffisamment marquées pour faire de la corrida à pied une véritable invention qui prend forme au milieu du XVIII siècle en Basse Andalousie ${ }^{15}$. Les innovations sont autant d'ordre formel que d'ordre socio-économique : la corrida s'est transformée en spectacle moderne. Il s'agit bien d'un changement de nature du divertissement

13 Callois, 1950, p. 130.

14 Sans que ces auteurs aient toujours les mêmes vues citons parmi d'autres Bennassar, 1993, Garcia Baquero, Romero de Solis, Vásquez Parlade, 1981, Guillaume Alonso, 1994, Saumade, 1994 et 1998.

15 Garcia Baquero, 1990 et Bennassar, 1993, chapitre 2 "L'invention de la tauromachie moderne ». pp. 33-52. 
taurin qui dans l'Espagne du milieu du XIX ${ }^{\mathrm{e}}$ siècle devient le spectacle de masse par excellence, réalité beaucoup plus tangible que l'appellation controversée de fiesta nacional. À cette rupture historique s'ajoute une rupture sociologique puisque la tauromachie moderne n'est plus envisagée dans la continuité de la tauromachie à cheval pratiquée par la noblesse mais comme l'aboutissement d'une forme urbaine et populaire autonome. Il se pourrait bien d'ailleurs que la corrida soit le premier spectacle de masse en Occident, en avance sur les spectacles sportifs modernes qui se développeront à une autre échelle à partir du $\mathrm{Xx}^{\mathrm{e}}$ siècle et dont le gigantisme des stades proclame une nouvelle étape ${ }^{16}$. Enfin, la corrida n'est plus le modèle festif unique, à l'aune duquel se mesure l'authenticité tauromachique et ses écarts. Il existe bien des pratiques tauromachiques plurielles, plus ou moins indépendantes les unes des autres, insérées dans des contextes festifs différents et dont l'unité ne peut être envisagée qu'à travers les processus de diffusion, d'acculturation, d'assimilation, les greffes et les rejets qui président à leur organisation.

\subsection{La modernité des pratiques tauromachiques}

Le $\mathrm{XIX}^{\mathrm{e}}$ siècle apparaît comme une phase déterminante durant laquelle les formes tauromachiques et leur répartition géographique acquièrent leurs principales caractéristiques. Trois phénomènes peuvent être mis en évidence : l'affirmation de la tauromachie professionnelle, la codification des pratiques tauromachiques régionales et la stabilisation des frontières des régions taurines.

Pour ce qui est de la corrida, au milieu du XVIII ${ }^{\mathrm{e}}$, la construction d'un édifice spécialisé ainsi que la séparation radicale des spectateurs et des professionnels sont acquises, même si la police montée est encore chargée de dégager la piste encombrée par la foule. Conformément à une conception wébérienne de l'État, l'édification de plazas de toros, situées dans l'idéal à l'extérieur de la ville et placées sous la surveillance des autorités peut alors être interprétée sinon comme l'aboutissement de la nécessité policière de conserver le monopole de la violence légitime tout au moins comme celui de contrôler au mieux «la multitude ». Quoi qu'il en soit, on constate une séparation entre d'un côté la fête taurine, communautaire et participative, et de l'autre le spectacle tauromachique professionnel et ordonné. À propos de la professionnalisation de la tauromachie, l'anthropologue Manuel Delgado Ruiz juge en outre très révélateur le souci constant de vouloir en perfectionner la valeur artistique. Considérer la tauromachie comme un art et non comme une fête, si l'on admet que l'art concerne d'abord le plaisir esthétique et intériorisé de celui qui en jouit, c'est l'assimiler à une activité qui n'est pas immédiatement fonctionnelle au niveau collectif ${ }^{17}$. En s'assurant d'une meilleure reconnaissance artistique auprès des élites, la tauromachie professionnelle perd donc en grande partie la fonction

16 Shubert, 2002, p. 25.

17 Delgado Ruiz, 1989, p. 32-38. 
sociale des festivités taurines populaires. Selon cet auteur, il s'agit d'une désactivation du rite tauromachique. Alors, la connivence toujours présente entre la fête et la tauromachie traduit peut être la volonté de conserver ou de recréer certaines vertus des jeux traditionnels auxquelles s'opposent, selon Elias et Dunning, les principes fondateurs du sport moderne qui émergent à la fin du XIX ${ }^{\mathrm{e}}$ siècle et dont la caractéristique est précisément de n’avoir « ni fonction rituelle, ni finalité festive $» 18$.

Le $\mathrm{XIX}^{\mathrm{e}}$ siècle est une période de diffusion et d'exportation de la corrida. Madrid, par sa centralité politique et géographique joue un rôle crucial de synthèse des tauromachies régionales puis de pôle de diffusion du modèle dominant: la corrida andalouse. On constate que l'adoption de la corrida espagnole dans le Sud-Ouest européen et en l'Amérique Latine est d'autant plus efficace qu'il existe dans ces régions des traditions tauromachiques autochtones. En France, la première «corrida à l'espagnole » a lieu à Bayonne en août 1853. Suivent la même année Mérignac, Nîmes, Arles, Marseille, Avignon puis sans grande continuité géographique Paris (1865), Périgueux (1866), Poitiers (1866), Le Havre (1868), Vichy (1892), Lyon (1894), Roubaix (1899), Nantes (1906), Saint Malo (1912). Le spectacle d'importation s'épanouit davantage dans la moitié Sud du pays, là où existent de longue date des traditions tauromachiques liées à la présence de troupeaux comme en Camargue et en Gascogne. Après une période de spectacles mixtes (courses hispano-landaises et hispano-provençales), les genres s'individualisent. Mais c'est en devenant un instrument de résistance des « libertés méridionales » face au centralisme jacobin qui multiplie les interdictions à l'endroit des pratiques taurines, que la corrida s'enracine véritablement à partir des années 1890. La loi Grammont de 1850 condamnant les mauvais traitements aux animaux domestiques est modifiée en 1951 et autorise officiellement la corrida avec mise à mort dans les municipalités qui peuvent se prévaloir «d'une tradition locale ininterrompue ${ }^{19}$.

En Amérique Latine, la situation est différente. Dès le XVI siècle, la conquête espagnole introduit aux Amériques du bétail pour approvisionner en viande les villes coloniales. À Lima et à Mexico, capitales de la Nouvelle Castille et de la Nouvelle Espagne, ainsi que dans bien d'autres villes récemment fondées, les fêtes tauromachiques de l'aristocratie se multiplient. Elles ont pour fonction de réjouir et d'édifier les populations récemment conquises par le spectacle de la noblesse en arme. En milieu rural, l'élevage bovin extensif devient rapidement une forme de contrôle et d'appropriation territoriale pour les classes dominantes. Ceci et l'ampleur du marronnage à l'échelle de l'immensité américaine donnent naissance à une très grande variété de jeux pratiqués par les populations indigènes, noires, métisses et créoles qui copient ou parodient l'usage des élites. La diversité des tauro-

18 Roger Chartier dans I'avant propos de Elias et Dunning, 1994, p. 15.

19 Pour ce qui concerne l'histoire de la corrida en France voir Lafront, 1977 et Bennassar, op. cit. 
machies américaines en est issue ${ }^{20}$. Cet héritage complexe a servi de relais à l'assimilation progressive de la corrida moderne dans les plus grandes villes telles que La Havane, Lima, Mexico, Buenos Aires, Montevideo, Bogota, La Paz, Quito, Panama qui accueillent les toreros de la Mère Patrie venus faire fortune aux Amériques. Certains États, au moment de leur indépendance, interdisent la corrida : l'Argentine en 1819, le Chili en 1823, l'Uruguay en 1890 et Cuba en 1898 sous autorité américaine. Cette condamnation a pour ambition politique de se débarrasser des symboles explicites de la domination coloniale, sans tenir compte des logiques complexes du métissage et de mettre un terme à une pratique considérée comme rétrograde par les élites progressistes.

Au contact de la corrida moderne ce sont donc l'ensemble des pratiques tauromachiques autochtones, existant du Sud-Ouest européen à l'Amérique Latine, qui en retour subissent des ajustements au fur et à mesure de sa diffusion. Frédéric Saumade, pour ce qui est des tauromachies européennes parle d'une «dynamique d'engendrement réciproque » ${ }^{21}$.

\subsection{Les festivités taurines d'aujourd'hui}

Les sociétés de protection des animaux et le rationalisme politique du $\mathrm{XIX}^{\mathrm{e}}$ siècle auraient volontiers fait disparaître les festivités taurines. Au XX $\mathrm{e}^{\mathrm{e}}$ siècle, on aurait pu penser que la mise en scène d'un affrontement réel entre l'homme et l'animal était condamnée à s'éteindre auprès de populations de plus en plus marquées par une urbanisation poussée des modes de vie où se mêlent à la fascination respectueuse pour la nature, les bons sentiments à l'égard de bêtes. On constate au contraire que les pratiques tauromachiques se maintiennent et pour certaines, connaissent un renouveau important.

Aussi paradoxal que cela puisse paraître pour certains, les fêtes taurines semblent parfaitement adaptées aux besoins et au fonctionnement des sociétés contemporaines qui les produisent : la récréation dont on assume désormais la portée re-créative, le développement économique dont on accepte qu'il puisse procéder d'activités apparemment non directement productives, l'affirmation des identités collectives qui ne cesse de valoriser l'authenticité de la culture et de la tradition. On trouve un bon exemple de l'adaptation des festivités taurines aux sociétés actuelles dans leur progressive institutionnalisation sur le modèle sportif. C'est le cas de la course landaise et de la course camarguaise dont les instances fédérales sont respectivement agrées en 1973 et 1975 par le Ministère de la Jeunesse et des Sports ${ }^{22}$. En Espagne, de nombreux adeptes des concours de recortadores sont également favorables à une institutionnalisation du même type ${ }^{23}$. Les

20 Sur l'introduction du bétail en Amérique Latine et la question du marronnage voir Deffontaines, 1957 et Digard, 1992.

21 Saumade, 1998, p. 178.

22 Puyo et Dauga, 2000, Dauga, 2002, Keerle, 2002, chapitre "Sportivisation, spatialisation, territorialisation : la course camarguaise» pp. 384-444.

23 Entretien personnel avec Alberto de Jesus, ancien champion d'Espagne de recortadores et directeur de la revue Bous al carrer. 
tauromachies américaines ont suivi un parcours comparable. Les activités de la charreria sont réglementées par l'Association Nationale des Charros depuis 1921 et reconnues sport national sous la tutelle de la Fédération Mexicaine de charreria depuis 1933. En 1969, la fédération vénézuélienne de coleo inscrite à l'Institut National du Sport regroupe toutes les associations du pays et en 1981, le Congrès National déclare le coleo "Sport criollo et traditionnel » 24 . La vaquejada, depuis le 3 mars 1998, est considéré comme « une pratique sportive et un spectacle populaire dans de nombreuses régions du pays dont l'importance économique, touristique et culturelle est croissante ${ }^{25}$. Il est intéressant de constater que la modernisation de la tauromachie par l'adoption des logiques d'organisation du sport a partout fait croître le nombre de spectacles et ne s'est pas réalisée en rupture avec les calendriers festifs traditionnels. En effet, les dates importantes des compétitions sportives continuent de coïncider en majorité avec les différentes fêtes des villes et des villages des régions taurines. Pour reprendre une observation de Dumazedier : « les fêtes traditionnelles [...] fixent un cadre rituel aux loisirs modernes, mais en même temps les loisirs modernes leur donnent un sens et un rôle nouveaux ${ }^{26}$.

Dans le cas de l'Espagne, l'augmentation générale du niveau de vie ainsi que l'urbanisation croissante du peuplement a largement contribué à la multiplication des spectacles du haut de la hiérarchie taurine. Cependant, cette augmentation n'a pas eu lieu au détriment des pratiques participatives dont le nombre ne fait que croître depuis une vingtaine d'années. En outre, on constate une tendance au regroupement des spectacles sur le modèle de la féria, un allongement des cycles festifs et la croissance de l'offre taurine pendant les mois de juillet et août en lien avec le développement du tourisme. À cet égard, les littoraux touristiques apparaissent comme des zones dynamiques où ont été construites de nombreuses arènes à partir des années 1960. Ce constat dépasse le cadre de l'Espagne comme en témoignent les arènes de la Albufeira, en Algarve, en tête des statistiques taurines portugaises ou les arènes de Cancun au Mexique qui de 1990 à 1993 ont programmé plus de 50 spectacles par an. Ceci étant, on peut considérer que l'adaptation des corridas à la modernité a été en partie différente des autres tauromachies professionnelles notamment en raison de sa dimension transnationale. D'une part, l'organisation des spectacles n'a pas suivi la voie d'un découpage des compétitions sur une base territoriale. D'autre part, la corrida n'a pas confié ses intérêts et son organisation à une quelconque autorité fédérative ou centralisatrice de type sportif ${ }^{27}$. L'organisation des spectacles dominée par l'initiative privée espagnole doit se conformer aux divers

24 FEVECO, fédération vénézuelienne de coleo. Le mot criollo, créole, désigne ici ce qui est autochtone.

25 Projet de loi ${ }^{\circ} 249$, Congresso Nacional.

26 Dumazedier, 1962, p. 64.

27 Prada, 1998. 
règlements taurins nationaux, régionaux ou municipaux dont les normes ne sont pas partout identiques ainsi qu'aux accords négociés entre professionnels. Par exemple, en Amérique latine, des conventions de type protectionniste, imposées par les syndicats de professionnels, obligent à une participation minimale de toreros nationaux lors des férias pour modérer la suprématie espagnole en matière d'exportation du spectacle.

Enfin, le renouveau des tauromachies participatives observé dans le cas du bous de carrer, des encierros ou des touradas a corda aux Açores semble directement lié au regain d'intérêt pour les traditions considérées comme identitaires et patrimoniales. En Espagne, il convient de rappeler que le franquisme avait officiellement interdit la pratique des festejos taurinos populares. Ils se déroulaient néanmoins dans la clandestinité, l'annonce d'une « exhibition de bétail » suffisant à rassembler les adeptes. Dans le contexte actuel d'une Espagne démocratique et d'une nouvelle répartition autonomique des compétences en matière de spectacles taurins, il apparaît clairement qu'une partie de la société civile trouve dans la tauromachie populaire un moyen d'expression en phase avec le développement des régionalismes. Ceci est d'autant plus remarquable qu'on avait cru la tauromachie définitivement en crise dans les années 1980 à cause de l'instrumentalisation franquiste de la corrida professionnelle.

\section{Tauromachies et identités territoriales}

\subsection{Autocélébration de la communauté et construction de l'identité}

Les approches anthropologiques de la tauromachie s'accordent à considérer qu'il existe une identification forte entre les protagonistes des divertissements taurins et les sociétés qui les produisent. Les taureaux et les hommes sont toujours célébrés en accord avec les valeurs qu'ils véhiculent au sein de chaque communauté de sorte que les fêtes taurines apparaissent comme un puissant vecteur identitaire et un moment privilégié de la reproduction sociale. Au-delà de ce dénominateur commun, il existe un large éventail d'interprétations de la tauromachie qui ne sont pas toutes compatibles entre elles : rite de passage, métaphore des rapports de genre, réminiscence archaïque d'un culte du taureau, ritualisation ludique de la conduite et de l'abattage des bovins, symbolisation des structures sociétales. À la vue des divergences d'interprétation, il n'est pas certain qu'une lecture totalisante et unificatrice de l'ensemble des pratiques tauromachiques soit tenable sans simplification outrancière.

La diversité géographique des pratiques taurines et leur fonctionnement en grande partie autonome incitent à relativiser les interprétations qui valorisent à l'excès la continuité des rites festifs taurins dans le temps et dans l'espace. Et si l'on fait de la tauromachie un rite, terme qui pourrait également être discuté, il nous semble peu convainquant qu'une quelconque ritualisation masque une signification immuable des fêtes taurines. Il nous 
paraît plus éclairant de montrer que les relations entre la fête et la tauromachie sont multiples, géographiquement variables et relèvent d'une « invention de la tradition » où convergent les intérêts identitaires et commerciaux. Cette tradition renvoie de la tauromachie l'image d'un phénomène très ancien, représentatif d'une culture authentique et caractéristique d'un territoire spécifique que la simple présence du taureau semble garantir et délimiter. Elle affirme aussi certain attachement à la terre et la ruralité remises à l'honneur par de nombreuses fêtes taurines (la féria des Vendanges à Nîmes, féria du Riz à Arles, fiesta del Olivo à Mora de Toledo, fiesta llanera au Venezuela). Il s'agit d'un mythe auquel se prête la passion taurine le temps de la fête. On y reconnaît la trace d'un discours identitaire essentialiste qui présente partout le taureau comme le symbole d'un peuple, le totem d'un territoire et parfois même comme l'objet d'un culte. Frédéric Saumade et Eric Baratay, le premier pour la course camarguaise, le second pour la corrida, ont retracé la construction historique de tels mythes 28 . Dans une approche constructiviste du fait taurin, il est intéressant de reconnaître l'efficacité de la fonction sociale de ces mythes qui ont largement participé à l'enracinement des traditions taurines dans le temps et dans l'espace.

\subsection{Fêtes taurines et expérience de l'altérité}

Les identités collectives exprimées par les fêtes taurines prennent toute leur signification lorsqu'elles sont mises en relation les unes avec les autres. Celles du voisin, proche ou lointain, sont toujours concurrentes et complémentaires. L'écrivain Bernard Manciet raconte que le public de Mont-deMarsan, insatisfait des bêtes et des écarteurs, n'a rien à crier de plus tendre que l'insulte : «à Dax, à Dax » ${ }^{29}$. Le jeu taurin peut explicitement reposer sur la rivalité des appartenances territoriales. C'est ce qui fonde en grande partie l'intérêt des compétitions internationales de coleo entre la Colombie et le Venezuela. À une autre échelle, le concours annuel de capeia raiana qui a lieu chaque année dans les arènes d'Aldea da Ponte, dans la région de Sabugal, constitue également un bon exemple. Le concours met en compétition les villages voisins. Chaque village est représenté par une équipe de trente individus qui affronte la charge d'un taureau par l'intermédiaire d'un forcao, une sorte de grande fourche en bois composée de pieux. La tauromachie semble exprimer directement la rivalité des identités locales. Dans le même temps, cette rencontre inter-villageoise matérialise une commune appartenance à la zone frontière, la raia. Autrefois, le taureau était volé du côté espagnol pour être vaincu en terre portugaise. La symbolique survit puisque le taureau est aujourd'hui acheté de l'autre côté de la frontière, dans les élevages de la province de Salamanque. Cette tra-

28 Baratay, 1997, Saumade, 1991.

29 Bernard Manciet dans I'avant propos de Valat, 1993, p. 14. Jacques, Pour la beauté du geste, la course landaise, Loubathière, Portet sur Garonne, p. 14, 1993. 
dition peut être interprétée comme la survivance d'une perception active de la frontière et de la rivalité territoriale entre les deux nations dans une zone historiquement marquée par de nombreuses incursions castillanes ${ }^{30}$.

On observe qu'au-delà des rivalités locales s'exprime souvent une complémentarité des oppositions qui révèle un sentiment d'appartenance commun d'un niveau supérieur. D'une certaine manière, les spectacles de coleo entre la Colombie et le Venezuela font exister le territoire du Llano, région naturelle et culturelle coupée en deux par les guerres d'indépendances du début du XIX siècle et restée en marge de l'intégration territoriale de ces deux pays rivaux. Dans un contexte différent, la course camarguaise fait exister la Camargue comme territoire d'appartenance entre Languedoc et Provence, de même que la course landaise fait exister un pays gascon entre Gers et Chalosse. La tauromachie participe incontestablement à la construction identitaire de ces territoires emboîtés. Nous pensons que l'adoption du modèle sportif rend très efficace la confrontation des identités spatiales dans la mesure où les festivités taurines se trouvent davantage reliées entre elles que s'il n'existait aucune forme de championnat à base territoriale. Elle favorise aussi l'articulation entre l'identité locale et les identités spatiales de rang supérieur. En outre, la victoire d'un protagoniste reconnue par des critères objectifs et identiques sur l'ensemble du territoire taurin constitue probablement un facteur de cohésion supplémentaire.

\subsection{La corrida : la clé de voûte d'une aire culturelle ?}

La corrida relève d'une territorialité en partie différente. Son centre est constitué par le doublet Madrid-Séville. Madrid représente le centre structurel, économique et décisionnel de la fiesta alors que Séville en représente plutôt le centre culturel et iconographique. La relation de concurrence indéniable entre les deux arènes établit également une relation de complémentarité à l'origine d'une véritable maîtrise de l'espace taurin. Ainsi la Castille et l'Andalousie forment un espace centre qui cumule les avantages comparatifs par rapport aux autres régions: les deux arènes de référence mondiale, un vivier inépuisable de toreros, les principaux élevages et l'immense majorité des membres du mundillo ${ }^{31}$. La relation des périphéries tauromachiques avec le centre varie alors en fonction de nombreux critères parmi lesquels la distance au centre et le niveau de dépendance extérieure pour l'organisation d'une féria. Certains pays d'Amérique Latine comme le Mexique ou la Colombie auraient les moyens d'être relativement indépendants du mundillo espagnol car ils possèdent des élevages et des toreros en nombre suffisant. Cependant, on observe de nombreuses alliances autour d'une offre tauromachique transcontinentale au bénéfice de l'Espagne qui exporte ses toreros.

30 Teixeira, 1995.

31 Le mundillo, le petit monde de la corrida, englobe les imprésarios, éleveurs, toreros, journalistes etc. 
À l'échelle de l'Espagne, certaines capitales régionales peuvent être considérées comme des centres secondaires. Les plus importantes sont situées à la périphérie de l'espace taurin espagnol. Au sein de chaque région autonome, la capitale constitue le centre décisionnel et identitaire de l'espace taurin régional: Pampelune en Navarre, Bilbao en Biscaye, Saragosse en Aragon, Valence dans la Comunidad Valenciana... Les férias de chacune de ces villes sont l'occasion d'exprimer des valeurs tauromachiques distinctes de la culture dominante. La volonté de se démarquer des valeurs dominantes de la tauromachie est rendue manifeste par l'introduction, pendant les férias, de pratiques tauromachiques régionales et locales. C'est le cas de Valence qui multiplie les concours de recortadores et qui réintroduit des pratiques tauromachiques locales et participatives au sein même des arènes de la capitale avec un objectif non dissimulé de patrimonialisation régionale de la culture taurine populaire. C'est aussi le cas plus ancien de Pampelune qui fait de l'encierro le symbole mondialement connu de sa féria. L'encierro peut alors être interprété comme l'expression d'une revendication identitaire en opposition avec le modèle tauromachique dominant. Il peut aussi être analysé comme un trait d'union entre deux cultures taurines, entre la tauromachie participative et la tauromachie professionnelle, entre l'identité régionale navarraise et l'identité nationale espagnole ${ }^{32}$. Dans le cas de la Catalogne, la tauromachie ou plutôt son rejet est l'instrument d'une différenciation plus radicale. Depuis une vingtaine d'années, les mesures qui tentent de faire disparaitre la culture taurine s'accélèrent. En 1988, une loi de protection des animaux limite la pratique des festejos populares et interdit la construction de nouvelles arènes. En avril 2004, le conseil municipal de Barcelone adopte une déclaration de rejet de la corrida par 21 voix contre 15 visant à faire interdire par le gouvernement autonome les pratiques tauromachiques en Catalogne. Un courant politique anti-taurin souhaite voir disparaitre un héritage jugé désormais incompatible avec une certaine conception de l'identité catalane. La tauromachie dans son ensemble constituerait le marqueur exclusif et politiquement incorrect de la nation espagnole dont certains veulent se débarrasser. Notons qu'à Céret de l'autre côté des Pyrénées, la tauromachie est au contraire un symbole fort de l'identité catalane. Enfin, dans le Sud-ouest et le Sud-est français, la pratique des courses landaises et des courses camarguaises sur un espace qui est commun à celui de la corrida relativise le monopole hispanique de la culture taurine.

Il est intéressant de constater que la corrida qui est perçue comme un signe de la culture espagnole peut en même temps être parfaitement assimilée au

32 Nous avons montré par ailleurs que le déroulement cyclique de la saison tauromachique a pour effet une circulation dans le temps et dans l'espace d'une centralité éphémère qui favorise d'autant la confrontation à distance des différentes cultures taurines ainsi que leur visibilité. Maudet, 2001. 
patrimoine de la culture locale, régionale voire nationale des pays taurins. Les couleurs des villes, des régions ou des nations qui flottent au-dessus des arènes en compagnie du drapeau espagnol en témoignent. En Amérique Latine, la corrida souligne un lien avec la Madre Patria (la Mère Patrie). Dans le Sud de la France, la corrida exprime une proximité culturelle avec l'Espagne et un fort sentiment d'hispanophilie. À titre d'hypothèse, le fait que la corrida relève davantage de la représentation artistique que du sport trouve un prolongement significatif. Nous pensons que le torero apparaît comme l'intercesseur d'une identification qui est différente de celle produite par le sportif dont l'exclusivité de l'appartenance territoriale est rendue plus explicite. La corrida combine un chauvinisme avéré et un principe d'intégration de l'altérité qui est soumis à la subjectivité du triomphe plus qu'à l'objectivité de la victoire. Lorsque vient à échouer un torero qui n'est pas du cru, il est renvoyé à son origine géographique qui signifie dans le même temps un déni de son talent. S'il triomphe, le public se reconnait en lui, quelle que soit son origine et le torero sort des arènes par la grande porte, ouverte sur la cité en signe d'adoption par la communauté. Le torero est comme naturalisé par le triomphe. C'est ainsi, parmi de très nombreux exemples, que Manolete, le grand matador espagnol des années 1940, originaire de Cordoue, devint "le torero de Lima ", de Mexico et d'ailleurs. C'est ainsi que César Rincon, colombien, devint au début des années 1990 "le torero de Madrid » acclamé au cri de «viva Colombia ». Lors de la féria de Quito en 2005, le public, pour encourager le torero national Guillermo Alban scandait «Ecuador, Ecuador, Ecuador ». La déroute du sévillan Morante de la Puebla s'est soldée par des « rentres chez toi, l'aéroport n'est pas loin » ${ }^{33}$. Le triomphe de Enrique Ponce, torero de Valence a motivé une forte ovation qui a même laissé échapper le cri «Ecuador, Ecuador » lorsque ce dernier, d'un geste symbolique, a ramassé une poignée de sable des arènes. Si cette hypothèse qui mériterait un plus ample développement se trouve confirmée, la corrida pourrait être considérée comme un marqueur territorial d'une hispanité syncrétique.

\section{Conclusion}

Les fêtes taurines sont des moments privilégiés d'affirmation des identités collectives dans un contexte de développement des loisirs et d'urbanisation croissante des modes de vie. Malgré la distinction entre la tauromachie professionnelle qui relève du spectacle et la tauromachie participative dont la dimension collective et intégratrice est directement perceptible, la tauromachie est vécue comme une fête qui assure une forte cohésion sociale et spatiale. On a vu que la professionnalisation des jeux taurins à la croisée du modèle sportif et de la représentation artistique ainsi que la réinvention permanente du lien entre la fête et le taureau peuvent être interprétées

33 Les arènes de Quito sont situées à quelques centaines de mètres de l'aéroport Mariscal Sucre. 
comme une adaptation de la tauromachie aux transformations des sociétés contemporaines. Tout se passe comme si les fêtes taurines garantissaient l'authenticité d'une identité territoriale qui revendique un enracinement historique ancestral et un attachement idéalisé à la ruralité. Il en résulte l'affirmation d'une aire culturelle au sein de laquelle les pratiques tauromachiques prennent formes et significations les unes par rapport aux autres à différentes échelles. D'un bout à l'autre de sa géographie, la tauromachie apparaît comme un critère commun de rapprochement et de différenciation territoriale où la perception d'une identité commune passe par l'expérience d'une altérité voisine ou radicale. Les fêtes taurines constituent ainsi un puissant marqueur territorial.

\author{
Jean-Baptiste Maudet \\ Casa de Velasquez, Calle de Paul Guirard, \\ 3 Ciudad Universitaria \\ 28040 Madrid Espagne \\ jb.maudet@wanadoo.fr
}

\title{
Bibliographie
}

Augustin J-P. (1995), Sport, Géographie et aménagement, Paris, Nathan, fac. Géographie.

Baratay E. (1997), "Comment se construit un mythe : la corrida en France au xxe siècle », Revue d'Histoire moderne et contemporaine, 44-2, pp. 305-330.

Bennassar B. (1993), Histoire de la tauromachie, une société du spectacle, Paris, Desjonquères.

Caillois R. (1950), L'homme et le sacré, Paris, Gallimard.

Dauga M. (2002), «L'âge d'or de la course landaise : I'adaptation d'une pratique culturelle à la modernité », Chalosse, l'esprit des lieux, entre mémoires et histoires, pp. 69-91.

Deffontaines P. (1957), "L'introduction du bétail en Amérique Latine », Les Cahiers d'Outre-Mer, $\mathrm{n}^{\circ} 37$, tome $\mathrm{X}$, pp. 5-22.

Delgado Ruiz M. (1989), «El toreo como arte o como se desactiva un rito », Taurologia, $n^{\circ} 1$, Madrid, pp. 32-38.

Digard J.-P. (1992), «Un aspect méconnu de I'histoire de l'Amérique: la domestication des animaux », L'Homme, 122-124, pp. 253-270.

Di Méo G. (2001), La géographie en fêtes, Paris, Géophrys.

Dumazedier J. (1962), Vers une civilisation du loisir ? Paris, Seuil.

Durand J. (1995), Humbles et Phénomènes, Éditions Verdier.

Elias N., Dunning E. (1994), Sport et civilisation, la violence maîtrisée, Paris Fayard.

Favory M. (2000), « Les bestiaires et l'espace, raisons géographiques de la passion taurine dans le sud-ouest européen », Sud-Ouest Européen, n 8, Toulouse, p. 5-14.

García-Baquero A., Romero de Solis P., Vasquez Parlade I. (1980), Sevilla y la fiesta de toros, Sevilla, Biblioteca de temas sevillanos.

García-Baquero A. (1990), "El macelo sevillano y los orígenes de la la tauromaquia moderna », Taurologia, $\mathrm{n}^{\circ} 2$, pp. 38-44.

Guillaume-Alonso A. (1994), La tauromaquia y su génesis, Bilbao, Ediciones Laga.

Keerle R. (2002), Sports et territoires, contribution à une géographie du pouvoir, Thèse doctorale de géographie sous la direction de M. Jean-Paul Volle, soutenue à l'université Paul Valéry - Montpellier III. 
Lafront A. (1977), Histoire de la corrida en France. Du Second Empire à nos jours, Paris Julliard

Maudet J.-B. (2003), "La organización funcional del espacio taurino. Apuntes de geografía taurina ", Fiesta de toros y sociedad, Universidad de Sevilla, pp. 490-494.

Maudet J.-B. (2006), « Tauromachie et géopolitique en péninsule lbérique : la frontière Espagne/Portugal depuis l'arène ", Mélanges de la Casa de Velázquez, 36 (1), pp. 259-281.

Prada J.I. de (1998), "Análisis comparado de la ordenación institucional de los toros y del deporte ", Revista de Documentación del Ministerio del Interior, $\mathrm{n}^{\circ} 17$.

Puyo J.-Y., Dauga M. (2000), " La course landaise, sport-spectacle confronté au défi de sa pérennisation (XIXe-Xxe siècle) ", Sud-Ouest Européen, $\mathrm{n}^{\circ}$ 8, Toulouse, p. 15-22.

Saumade F. (1991), "Mythe et histoire dans une société du spectacle tauromachique », Ethnologie française, 2, tome XXI, pp. 148-159.

Saumade F. (1994) Des sauvages en Occident. Les cultures tauromachiques en Camargue et en Andalousie, Paris, M.S.H.

Saumade F. (1998), Les tauromachies européennes, Paris, Éditions du C.T.H.C.

Shubert A. (2002) A la cinco de la tarde, una historia social del toreo, Madrid, Turner.

Teixeira F. (1995), «A corrida do forçao », Mediterrâneo, Lisboa, n 5/6, pp. 23-32.

Traimond B. (1996), Les fêtes du Taureau, AA Éditions, Bordeaux.

Valat J. (1993), Pour la beauté du geste, la course landaise, Loubathière, Portet sur Garonne. 